\title{
Spontaneous mesenteric haөmatoma: a complication of oral anticoagulant use
}

Singapore Med J 2021; 62(9): 504-506 https://doi.org/10.11622/smedj.2021150

Dear Sir,

Spontaneous mesenteric haematoma $(\mathrm{SMH})$ is a rare condition, with only 110 cases reported in the $20^{\text {th }}$ century. ${ }^{(1)}$ In recent years, a few cases of SMH secondary to oral anticoagulant use have been reported.(2,3) Approval of the use of novel oral anticoagulants (NOACs) for atrial fibrillation in the last decade has increased the number of therapeutic alternatives to warfarin and may have led to the increased prescription of anticoagulants owing to their more favourable pharmacokinetic profile. ${ }^{(4)}$ We herein report on two cases of SMH in our institution. Both patients were on NOACs for atrial fibrillation and presented with abdominal pain and haemodynamic instability. One of the patients was managed with angioembolisation, while the other required emergency laparotomy.

In Case 1, a 60-year-old woman with a significant medical history of atrial fibrillation for which she was taking rivaroxaban presented with acute onset of generalised abdominal pain that was associated with non-bilious, non-bloody vomiting. Clinical examination revealed a drowsy patient with generalised abdominal tenderness. Further investigation showed lactic acidosis and an acute drop in haemoglobin from $15 \mathrm{~g} / \mathrm{dL}$ to $9 \mathrm{~g} / \mathrm{dL}$. The coagulation profile of the patient was deranged, with a raised prothrombin time (PT) of 26.1 seconds and an international normalised ratio (INR) of 2.6. Computed tomography (CT) of the abdomen and pelvis (Figs. 1a \& b) showed a large $22 \mathrm{~cm} \times 20 \mathrm{~cm}$ intra-abdominal haematoma with contrast extravasation; however, the culprit vessel could not be identified. Additional tests were performed in consultation with a haematologist. Disseminated intravascular coagulopathy (DIVC) screening showed normal fibrinogen with mildly raised D-dimer, which was not suggestive of DIVC. Antifactor Xa assay is not recommended in this emergency setting, as it requires several hours for processing. Unfortunately, the patient became increasingly unstable and developed fast atrial fibrillation. She was started on inotropic support and amiodarone infusion, and the massive transfusion protocol was activated.

A discussion was held with the interventional radiologist (IR), and it was decided to proceed for exploratory laparotomy instead of angioembolisation, as the culprit vessel could not be identified on imaging. The patient was scheduled for emergency exploratory laparotomy, bearing in mind the possible need for on-table angiography. Preoperative consideration included the use of a leadlined operating theatre, fluoroscopy-compatible operating table and constant communication with the IR to reduce time lost upon activation. However, the patient deteriorated on the table with a circulatory collapse and pulseless electrical activity after induction, likely owing to loss of tone. Cardiopulmonary resuscitation was commenced, with successful return of spontaneous circulation after resuscitation. A discussion was held with the anaesthetist and the vascular surgeon. Given the high surgical risk and the possibility that laparotomy might cause the large mesenteric haematoma to further lose its tamponade effect, leading to worsening haemodynamic instability and bleeding, urgent on-table angioembolisation was decided. Constant communication with the IR helped to reduce the time to intervention. While waiting for the IR to arrive, the anaesthetists aggressively resuscitated the patient; she was relatively stable by the time the IR arrived, and angioembolisation was commenced promptly, with minimal delay. Fortunately, the bleeding vessel was identified to arise from a branch of the superior mesenteric artery (SMA), which was angioembolised with glue. The post-embolisation recovery of the patient was complicated by acute renal failure requiring dialysis and an infected haematoma that required laparotomy for evacuation of the infected hematoma and washout. Her renal function eventually returned to baseline and she was discharged well 45 days later.

In Case 2, an 83-year-old woman with ischaemic heart disease and atrial fibrillation that was being treated with apixaban presented with acute onset of abdominal pain; she denied any trauma prior to admission. On examination, the patient was hemodynamically stable and had mild distension over the lower abdomen, but no tenderness or guarding was noted. Investigations showed an acute
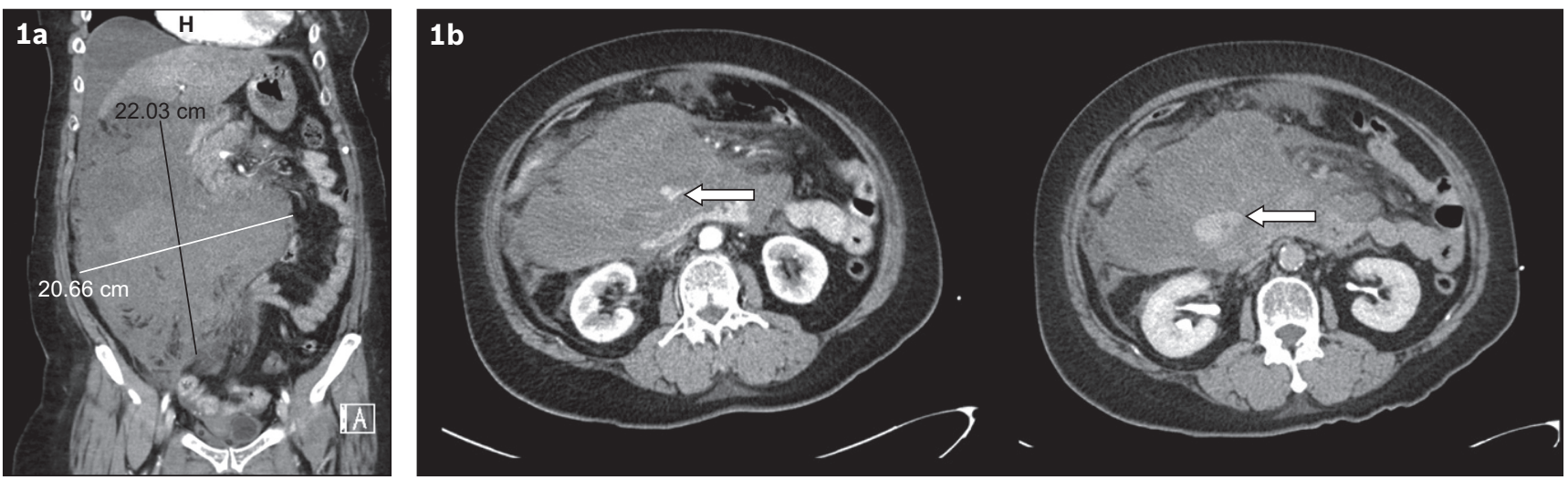

Fig. 1 (a) CT image of the abdomen and pelvis in coronal view shows a large intra-abdominal hematoma measuring $22 \mathrm{~cm} \times 20 \mathrm{~cm}$ in the right side of the abdomen. (b) CT images in axial view show the arterial phase (left) and delayed phase (right). An arterial contrast 'blush' (arrows) can be appreciated in the arterial phase, with delayed contrast pooling on the delayed phase, consistent with active haemorrhage. 
drop of haemoglobin level from $12.2 \mathrm{~g} / \mathrm{dL}$ to $10.5 \mathrm{~g} / \mathrm{dL}$. CT of the abdomen and pelvis revealed haemoperitoneum centred around the spleen, with no definite splenic laceration or 'blush' observed. Given that the patient was hemodynamically stable and clinically well, and the CT did not show an active 'blush', a decision was made for conservative management with serial trending of haemoglobin.

The patient became increasingly unstable and unresponsive to fluids and underwent an emergency exploratory laparotomy after she was adequately resuscitated. Intraoperative findings revealed $1.5 \mathrm{~L}$ of hemoperitoneum from a mesenteric tear near the splenic flexure mesentery. The unhealthy splenic flexure was resected, and the patient had a temporary abdominal closure following the principles of damage control. Anastomosis of the colon was performed at the subsequent relook laparotomy, in which the proximal and distal colonic stumps appeared healthy. A splenectomy was also performed for persistent oozing from the splenic hilum. The patient's recovery was complicated by colic-colic anastomotic breakdown requiring an end colostomy. She also developed enterocutaneous fistula at the proximal jejunum, which resulted in high fistula output. This was managed with insertion of a feeding tube through a percutaneous endoscopic gastrostomy (PEG) into the enterocutaneous fistula, and the patient was commenced on PEG feeding with concurrent oral comfort feeding. She was discharged from the hospital seven months after surgery.

Mesenteric haematoma is a rare condition, and SMH is even more uncommon, ${ }^{(5)}$ with most evidence in literature limited to case reports or series. The exact definition of SMH is unclear. Reilly et al considered SMHs to be haematomas that occur in the absence of any aetiology such as trauma, vascular disorders or pregnancy. ${ }^{(6)}$ However, Liang et al considered SMHs as those occurring in patients with risk factors such as connective tissue disorders, coagulopathies and those without any identifiable cause (idiopathic). ${ }^{(7)}$ Hirano et al further classified mesenteric haematomas into traumatic and non-traumatic - which are associated with mesenteric vascular diseases such as vasculitis - and spontaneous, which may be associated with anticoagulation therapy, connective tissue disease and other diseases such as Crohn's disease. Further clarity on the exact definition of mesenteric haematomas is warranted. The risk factors for mesenteric haematomas include pancreatitis, ${ }^{(8)}$ connective tissue disorders, ${ }^{(9)}$ Crohn's disease, ${ }^{(10)}$ haemophilia, ${ }^{(11)}$ incarcerated inguinal hernias, ${ }^{(12)}$ and antiplatelet and anticoagulation medications. Patients without any risk factors for mesenteric haematomas should be identified as having spontaneous haematomas.

Not many cases of SMH associated with anticoagulant use have been reported. Aoki et al ${ }^{(13)}$ reported ten cases of SMH. Liang et $\mathrm{al}^{(7)}$ reported another case series of 20 patients with $\mathrm{SMH}, 17$ of whom were diagnosed after the publication of the paper by Aoki et al. In the paper by Liang et al, five of the patients had been on warfarin and two had been on aspirin. However, only one case of SMH associated with NOAC use has been reported so far, by Hirano et al. ${ }^{(2)}$ In this case, a tear in the sigmoid colon mesentery resulted in bleeding and subsequent formation of mesenteric haematoma. This is similar to the presentation of our patient in Case 2.

Diagnosis is often made by CT imaging, with the initial intention being evaluation of the cause of acute abdominal pain. CT imaging also has the benefit of identifying possible causes such as pancreatitis, and complications including bowel obstruction and ischaemia. Where possible, CT angiography may provide additional information such as identification of the culprit vessel and evidence of active bleeding or 'blush'.

Initial management includes close haemodynamic monitoring, evaluation for complications and correction of coagulopathy. Unfortunately, unlike warfarin, quantitative tests for measurement of NOACs are not widely available. The use of quantitative thrombin levels and anti-Xa assay may provide the most accurate status of the anticoagulation effect of dabigatran and direct Xa inhibitors, respectively. However, the limited availability of these tests in only specialised laboratories and the long turnaround time limit their use in emergencies. Surrogate markers such as thrombin time and activated partial thromboplastin time can be used for dabigatran, and PT and INR can be used for anti-Xa inhibitors. ${ }^{(14)}$ Reversal agents such as andexanet and idarucizumab are relatively new drugs that have been approved for use by the United States Food and Drug Administration. However, idarucizumab is available in Singapore.

The definitive management of SMH depends on the haemodynamic stability of the patient, the size of the haematoma, evidence of active bleeding, and complications such as infection or mass effect. Conservative management can be considered in stable patients who are asymptomatic or have relatively small haematoma. Repeat imaging should be performed at a later interval to rule out complications and look for resolution of the haematoma. ${ }^{(1,5)}$ The classical intervention is surgery to evacuate haematomas and achieve haemostasis. ${ }^{(6)}$ However, care should be taken during induction and upon initial incision, as sudden loss of tone and decompression can precipitate cardiovascular collapse, as in Case 1. Preparation should be similar to that required for ruptured aortic aneurysm, whereby close communication with the anaesthetist is necessary. The surgeon should be gowned and ready, and the patient's abdomen should be prepped before induction of anaesthesia, with plans for clamping of aorta in the shortest possible time in case of cardiovascular compromise. At present, angioembolisation is an alternative to achieve haemostasis. In particular, angioembolisation should be considered in patients with evidence of active bleeding but without complications such as infection. However, successful angioembolisation surgery may still be warranted if complications such as infection of haematoma or bowel ischemia develop later. ${ }^{(2)}$ While the patient in Case 2 may have benefited from IR intervention as opposed to laparotomy at the outset, the lack of a 'blush' on the CT image to guide the proceduralist as to which vessel to intervene on may lead to higher rates of failure and also a potentially longer procedure. On hindsight, the unhealthy colon that was not observed on CT imaging would have eventually perforated and necessitated a laparotomy.

Complications that can arise from spontaneous mesenteric haematoma include partial or complete bowel obstruction, bowel ischaemia secondary to compromised blood supply within the mesentery, ${ }^{(7,8)}$ and infection of haematoma. 
In the first case, angioembolisation rather than surgery was performed, although the initial intent was exploratory laparotomy. However, experiencing a cardiovascular collapse on table prior to surgery rendered the surgery high risk. In this case, adoption of principles of damage control surgery with abbreviated surgical procedures limited to haemorrhage control and correction of physiological derangements of acidosis and coagulopathy becomes a priority. In most cases, safe surgical principle dictates an upfront surgical intervention rather than angioembolisation for patients in extremis. In this case, a decision was made to proceed with on-table angioembolisation instead of surgery, as the patient had already suffered a cardiovascular collapse, likely owing to loss of tone upon induction. Persisting with laparotomy would likely have had a catastrophic effect, with loss of tamponade effect caused by decompression of the haematoma during laparotomy.

In the second case, the site of bleeding was identified preoperatively on imaging. Laparotomy was performed to stop the bleeding that was causing a hypovolaemic shock, which was only transiently responsive to fluid. Laparotomy has the additional benefit of allowing the large blood clot to be evacuated to reduce the chance of secondary clot infection and allow bowel complications to be assessed. In this case, the splenic flexure colon was unhealthy and had to be resected in the same setting.

In conclusion, spontaneous mesenteric haematoma is a rare but serious clinical condition that should be considered in a patient on anticoagulants who presents with acute abdominal pain. The use of anticoagulants increases the risk of persistent bleeding and haematoma expansion. Hence, early interventions with surgery or angioembolisation should be considered in unstable patients. Stable patients who are suitable for conservative management should be closely monitored for delayed complications or haemodynamic compromise.

Yours sincerely,

Sheena Say Hoon $\underline{\text { Phua }}^{1}$, Saleem $\underline{\text { Ahmed }}^{1}$, Sundeep $\underline{\text { Punamiya }}{ }^{2}$, Kaushal Amit $\underline{\text { Sanghvi }}^{1}$

${ }^{1}$ Department of General Surgery, ${ }^{2}$ Department of Vascular and Interventional Radiology, Tan Tock Seng Hospital, Singapore. sheena.phua@mohh.com.sg

\section{References}

1. Parker SG, Thompson JN. Spontaneous mesenteric haematoma; diagnosis and management. BMJ Case Rep 2012; 2012:bcr2012006624.

2. Hirano K, Bando T, Osawa S, et al. Spontaneous mesenteric hematoma of the sigmoid colon associated with rivaroxaban: a case report. Int J Surg Case Rep 2018; 44:33-7.

3. Hosseini M, Hosseinzadeh A, Raoufian K, Hedjazi A. Nontraumatic retroperitoneal hematoma after warfarin administration: fatal case report and review of the literature. Am J Forensic Med Pathol 2015; 36:245-8.

4. Khan F, Huang H, Datta YH. Direct oral anticoagulant use and the incidence of bleeding in the very elderly with atrial fibrillation. J Thromb Thrombolysis 2016; 42:573-8.

5. Abbasi D, Vanhook JE, Salartash K, Levite H. Diagnosis and management of rare case of mesenteric hematoma rupture after transcatheter aortic valve replacement (TAVR): a case report and review of the literature. Case Rep Vasc Med 2018; 2018:6273538.

6. Reilly EF, Blansfield JA, Zebley DM. Spontaneous colonic mesenteric hemorrhage: report of an unusual case of abdominal apoplexy. Dis Colon Rectum 2005; 48:1484-6.

7. Liang C, Takahashi K, Furuya K, et al. Spontaneous mesenteric hematoma after antiplatelet therapy: a case report. Int Surg Journal 2018; 5:2349-52.

8. Toyonaga T, Nagaoka S, Ouchida K, et al. Case of a bleeding pseudoaneurysm of the middle colic artery complicating acute pancreatitis. Hepatogastroenterology $2002 ; 49: 1141-3$.

9. Hosaka A, Miyata T, Shigematsu H, et al. Spontaneous mesenteric hemorrhage associated with Ehlers-Danlos syndrome. J Gastrointest Surg 2006; 10:583-5.

10. Ashrafian H, Manfield JHR, Mitra A, Boyle DJ, Mathur P. Spontaneous mesenteric hematoma complicating an exacerbation of Crohn's disease: report of a case. BMC Surg 2014; $14: 35$.

11. Prasad S, Patankar T, Krishnan A, Pathare A. Spontaneous isolated lesser sac hematoma in a patient with hemophilia. Indian J Gastroenterol 1999; 18:38-9.

12. Sutton E, Emuakhagbon V, Morgan Jones V, McMasters K. Spontaneous mesenteric hematoma associated with recurrent incarcerated inguinal hernia. Am Surg 2015; $81:$ E3-5.

13. Aoki T, Nishizono M, Niina H, et al. A case of spontaneous mesenteric hematoma and a review of 17 cases in Japan. Gastroenterol Jpn 1990; $25: 768-73$.

14. Samuelson BT, Cuker A, Siegal DM, Crowther M, Garcia DA. Laboratory assessment of the anticoagulant activity of direct oral anticoagulants: a systematic review. Chest 2017; 151:127-38. 